\title{
Extent of neck dissection for patients with clinical N1 oral cancer
}

\author{
Yasumasa Kakei ${ }^{1} \cdot$ Hirokazu Komatsu $^{2} \cdot$ Tsutomu Minamikawa $^{1} \cdot$ Takumi Hasegawa $^{1} \cdot$ Masanori Teshima $^{2}$. \\ Hirotaka Shinomiya ${ }^{2} \cdot$ Naoki Otsuki $^{2} \cdot K^{2}$-ichi Nibu ${ }^{2}$ (1) Masaya Akashi ${ }^{1}$
}

Received: 9 October 2019 / Accepted: 12 February 2020 / Published online: 5 March 2020

(c) The Author(s) 2020

\begin{abstract}
Background No clear consensus has been reached on the indication of supraomohyoid neck dissection (SOHND) for clinically positive lymph-node metastasis.

Patients Consecutive 100 patients with previously untreated oral cancer treated at Kobe University Hospital were included in this study. All patients were clinically staged as anyTN1M0 and underwent radical dissection of the primary site and level I-V neck dissection as the initial treatment.

Results None of the 100 patients had pathological lymph-node metastasis (pLN) to level V. pLN to level IV was observed in two patients with tongue cancer in whom clinical lymph-node metastasis was preoperatively observed at level II.

Conclusions Level V may be excluded in the neck dissection for patients with N1 oral cancers. Level IV dissection should be considered in the patient with tongue cancer and clinical lymph-node metastasis at level II.
\end{abstract}

Keywords Tongue cancer · Clinical N1 · Neck dissection · Level IV · Level V · Supraomohyoid neck dissection · N1 · Oral cancer

\section{Introduction}

Supraomohyoid neck dissection (SOHND) is classified as a selective dissection of levels I, II, and III of the neck [1]. Since skip metastasis to level IV is rare in oral cancer [2] and recent advances in diagnostic imaging including enhanced computed tomography (CT), magnetic resonance imaging (MRI), as well as positron emission tomography/ computed tomography (PET-CT) have provided precise preoperative evaluation of neck lymph-node metastases, a prospective trial on prophylactic neck dissections in oral cancer was conducted and found no significant difference in survival between modified radical neck dissection (MRND) and SOHND groups [3]. Now, SOHND has been accepted worldwide as a technique of prophylactic neck dissection

Yasumasa Kakei and Hirokazu Komatsu have contributed equally to this article.

Ken-ichi Nibu

nibu@med.kobe-u.ac.jp

1 Department of Oral and Maxillofacial Surgery, Kobe University Graduate School of Medicine, Kobe, Japan

2 Department of Otolaryngology, Kobe University Graduate School of Medicine, Kobe, Japan for high-risk clinical N0 (cN0) tongue/oral cancers $[4,5]$. Furthermore, sentinel node biopsy (SNB) has been gradually accepted as a reliable staging test for patients with early disease and radiologically N0 neck. Since SNB can detect occult metastases with a sensitivity of 86-94\%, patients with no sign of metastases on SNB could avoid neck dissection [6].

On the other hand, no clear consensus has been reached on the clinical N1 (cN1) cases, although several studies recommended SOHND even for clinical N1 (cN1) cases in recent reports $[7,8]$. To explore the optimal surgical procedure for $\mathrm{cN} 1$ oral cancers, in this study, we investigated the appropriateness of SOHND in patients with $\mathrm{cN} 1$ oral cancer through a retrospective review of pathological neck lymphnode metastases (pLN) among the patients who underwent level I-V neck dissection for the treatment of $\mathrm{cN} 1$ oral cancer at Kobe University Hospital.

\section{Materials and methods}

This retrospective study included consecutive 100 patients with previously untreated oral squamous cell carcinoma, who were treated at Department of Oral and Maxillofacial 
Surgery or Department of Otolaryngology-Head and Neck Surgery, Kobe University Hospital, between January 1999 and December 2016. All the patients were preoperatively evaluated by CT and MRI. PET-CT was also employed in most cases. Ultrasound (US)-guided fine needle aspiration biopsy (FNAB) was performed as an additional examination when judging was difficult by CT, MRI, and PET-CT. Diagnostic criteria used for CT, MRI, PET-CT, and US in this study were described elsewhere $[9,10]$. Cancers were clinically staged as anyTN1M0 according to the seventh edition of TNM Classification of Malignant Tumours (UICC), and underwent radical resection of the primary site and level I-V neck dissection as the first-line treatment. Patients underwent postoperative radiotherapy with or without cisplatin when patients were at high risks such as positive or close surgical margins, extranodal extension, or multiple lymphnode metastases. Patients were followed up at our outpatient clinic, monthly in the first year, bimonthly in the second year, and then trimonthly. In general, patients were evaluated by CT every 6 months and by PET-CT every year.

The patients' ages ranged from 31 to 88 years (average, 68 years), with 58 males and 42 females. Most common primary site was tongue (45 patients), followed by lower gingiva (24), buccal mucosal (15), oral floor (8), and upper gingiva (8). As for T classification, 4 patients were classified as T1, 49 as T2, 22 as T3, and 25 as T4. Clinical and pathological data were obtained from medical records of the patients. Clinical distribution and pathological distribution of the lymph-node metastasis were evaluated according to the characteristics of the patients. This study was approved by the ethical committee of Kobe University Hospital
(\#1401) and written informed consent was obtained from all the patients.

\section{Results}

\section{Preoperative level of clinical lymph-node metastasis}

The preoperative levels of clinical lymph-node metastasis (cLN) are summarized in Table 1. Before surgery, cLN was observed at level I or II in all patients. No patients had cLN at level III, IV, or V. Among the patients with tongue cancer, metastasis to level II was common, observed in 25 (56\%) of the 45 patients. Among the patients with buccal mucosal, oral floor, or gingival cancer, cLN was observed at level IB in $43(78 \%)$ of the 55 patients, and only $12(22 \%)$ had cLN at level II.

\section{Pathological lymph-node metastases and subsite of metastasis}

At least one pLN was observed in 66 of the 100 patients. Thirty-seven patients had one pN (pLN1), and 29 had multiple pLNs (pN2b). The levels of the pLN farthest from the primary subsite (most distal metastasis) in individual patients are summarized in Table 2. In most patients, the most distal metastasis was at level III or nearer. Only two patients with pN2b had pLN at level IV, and both of them also had metastatic lymph nodes at level III. Metastasis to level $\mathrm{V}$ was not observed in any patient of this study.
Table 1 Level of clinical lymph-node metastasis by primary subsite

\begin{tabular}{lccccccc}
\hline & No. of pts & IA & IB & II & III & IV & V \\
\hline Tongue & 45 & 2 & 18 & 25 & 0 & 0 & 0 \\
Lower gingiva & 24 & 0 & 20 & 4 & 0 & 0 & 0 \\
Buccal mucosa & 15 & 0 & 12 & 3 & 0 & 0 & 0 \\
Oral floor & 8 & 0 & 5 & 3 & 0 & 0 & 0 \\
Upper gingiva & 8 & 0 & 6 & 2 & 0 & 0 & 0 \\
Total & 100 & 2 & 61 & 37 & 0 & 0 & 0 \\
\hline
\end{tabular}

No number, Pts patients, $I A-V$ levels IA-V of the neck

\begin{tabular}{lllrllll}
\hline & No. of pts & IA & IB & II & III & IV & V \\
\hline pN1 & 37 & 2 & 20 & 14 & 1 & 0 & 0 \\
pN2b & 29 & 1 & 8 & 10 & 8 & 2 & 0 \\
Total & 66 & 3 & 28 & 24 & 9 & 2 & 0 \\
\hline
\end{tabular}

No number, Pts patients, $I A-V$ levels IA-V of the neck, $p N 1$ one pathological lymph-node metastasis, $p N 2 b$ two or more pathological lymph-node metastases

*Based on the most distal level where lymph-node metastasis was pathologically identified in individual patients 


\section{Level of pathological lymph-node metastasis according to the primary subsite}

For the 66 patients who had pLN(s), the most distal levels of pLN were summarized by primary subsite in Table 3 . Metastasis to level IV was observed only in two patients with tongue cancer, while the pLN of all other cancers were limited to level I-III. There was no significant difference in the number of pLNs between subsites or T stages (Table 4).

\section{Levels of clinical and pathological lymph-mode metastases}

Among the patients with cLN in level I, the pLN(s) was limited to level I or II in 38 (90\%) of the 42 subjects. Only four patients (10\%) had metastasis at level III, and none at level IV. As for the patients with cLN to level II, five (21\%) and two $(0.8 \%)$ had pLN at level III and level IV, respectively (Table 5).

\section{Discussion}

Andersen et al. performed selective neck dissection for 106 oral cancers with $\mathrm{cLN}(\mathrm{s})$ including 58 patients with $\mathrm{cN} 1$. With the positive results of a 5-year disease-specific survival of $88.1 \%$, a 5 -year failure rate in the neck of $6.7 \%$, and a low regional recurrence rate of $4.3 \%$, they concluded that selective neck dissection is useful even in patients with $\operatorname{cLN}(\mathrm{s})$, depending on the size and mobility of the lymph
Table 3 Distribution of pathological lymph-node metastases by primary subsite
Table 4 Distribution of pathological lymph-node metastases by clinical $t$ stage
Table 5 Distribution of pathological lymph-node metastases by the level of clinical lymph-node metastasis

\begin{tabular}{lrrrrrrrrr}
\hline & $\mathrm{pN}+$ & $\mathrm{pN} 1$ & $\mathrm{pN} 2 \mathrm{~b}$ & $\mathrm{IA}$ & $\mathrm{IB}$ & $\mathrm{II}$ & $\mathrm{III}$ & $\mathrm{IV}$ & $\mathrm{V}$ \\
\hline Tongue & $32 / 45(71 \%)$ & 18 & 14 & 1 & 10 & 14 & 5 & 2 & 0 \\
Lower gingiva & $11 / 24(46 \%)$ & 4 & 7 & 0 & 7 & 4 & 0 & 0 & 0 \\
Buccal mucosa & $11 / 15(73 \%)$ & 7 & 4 & 2 & 6 & 3 & 0 & 0 & 0 \\
Oral floor & $5 / 8(63 \%)$ & 3 & 2 & 0 & 1 & 1 & 3 & 0 & 0 \\
Upper gingiva & $7 / 8(88 \%)$ & 5 & 2 & 0 & 4 & 2 & 1 & 0 & 0 \\
Total & $66 / 100(66 \%)$ & 37 & 29 & 3 & 28 & 24 & 9 & 2 & 0 \\
\hline
\end{tabular}

$p N+$ pathological lymph-node positive, $p N 1$ one pathological lymph-node metastasis, $p N 2$ two pathological lymph-node metastases, $I A-V$ levels IA to $\mathrm{V}$ of the neck

*Based on the most distal level where a lymph-node metastasis was pathologically identified in individual patients

\begin{tabular}{lcrrrrrrrr}
\hline & $\mathrm{pN}+$ & $\mathrm{pN} 1$ & $\mathrm{pN} 2 \mathrm{~b}$ & $\mathrm{IA}$ & $\mathrm{IB}$ & $\mathrm{II}$ & $\mathrm{III}$ & $\mathrm{IV}$ & $\mathrm{V}$ \\
\hline $\mathrm{T} 1$ & $4 / 4(100 \%)$ & 4 & 0 & 0 & 3 & 1 & 0 & 0 & 0 \\
$\mathrm{~T} 2$ & $39 / 49(80 \%)$ & 17 & 19 & 2 & 15 & 11 & 6 & 2 & 0 \\
$\mathrm{~T} 3$ & $14 / 22(64 \%)$ & 7 & 7 & 0 & 4 & 8 & 2 & 0 & 0 \\
T4 & $11 / 25(44 \%)$ & 9 & 3 & 1 & 6 & 4 & 1 & 0 & 0 \\
Total & $66 / 100(66 \%)$ & 37 & 29 & 3 & 28 & 24 & 9 & 2 & 0 \\
\hline
\end{tabular}

$p N+$ pathological lymph-node positive, $p N 1$ one pathological lymph-node metastasis, $p N 2$ two pathological lymph-node metastases, $I A-V$ levels IA to $\mathrm{V}$ of the neck

*Based on the most distal level where a lymph-node metastasis was pathologically identified in individual patients

\begin{tabular}{llllllll}
\hline \multirow{2}{*}{$\begin{array}{l}\text { Clinical LN metas- } \\
\text { tasis }\end{array}$} & \multicolumn{9}{l}{ Pathological LN metastasis } & & & & \\
\cline { 2 - 8 } & Total & IA & IB & II & III & IV & V \\
\hline Level I & $42 / 63$ & 3 & 25 & 10 & 4 & 0 & 0 \\
Level II & $24 / 37$ & 0 & 3 & 14 & 5 & 2 & 0 \\
Total & $66 / 100$ & 3 & 28 & 24 & 9 & 2 & 0 \\
\hline
\end{tabular}

$L N$ lymph node, $I A-V$ levels IA to $\mathrm{V}$ of the neck

*Based on the most distal level where a lymph-node metastasis was pathologically identified in individual patients 
nodes and histories of prior neck surgery and/or radiotherapy [11]. Kowalski et al. [7] and Asakage et al. [8] have also acknowledged SOHND as an appropriate treatment option for N1 patients whose metastasis is limited to level I because of the low frequencies of metastasis to level IV between 0 and $0.6 \%$. However, Shah et al. have reported that $15-16 \%$ of tongue/oral cancer with cLN(s) had pLN(s) to level IV [2], and many experts take a cautious stance on limiting (sparing) the extent of dissection. Suzuki et al. [12] recommended extended SOHND, which includes dissecting level $\mathrm{IV}$, since 1 of the 52 patients with $\mathrm{cN} 1$ tongue cancer had $\mathrm{pLN}$ at level IV. Harada et al. examined 95 patients with oral cancer, with only one of them, a patient with tongue cancer, experiencing metastasis to level IV. As in our present study, oral cancer originating in other subsites did not spread to level IV [13]. These results suggest that in the treatment of anyTN1 oral cancer except of tongue, level IV dissection can be excluded, whereas when treating tongue cancer, we should be aware that metastasis to level IV can occur, although rare in frequency.

In the present study, two patients with tongue cancer who had pLN in level IV were preoperatively diagnosed as having cLN at level II. Extended SOHND including level IV should be considered, at least in the treatment of tongue cancer with cLN at level II. Shimbashi et al. have reported that 2 out of 22 patients with tongue cancer had pLN to level IV and, similar to our present results, both had multiple pLNs [14]. Accordingly, in our study, metastasis to level IV was accompanied by pLN in level III in both patients, without the so-called skip metastasis [15]. As proposed by Koerdt et al. [16], a strategy may be successful to consider dissecting level IV if metastatic lymph node at level II or III is identified by intraoperative frozen section.

Similar to reports from Japan and overseas [8, 12], pLN to level V was not observed among our patients either. Recent meta-analysis by Liang et al. found no significant difference between selective and radical neck dissection in terms of regional control and prognosis [17], suggesting a little impact of level $\mathrm{V}$ dissection on the prognosis of the patients with oral cancer. Moreover, avoiding the dissection of level $\mathrm{V}$ reduces postoperative damage to the accessory nerve [19]. Taken together, standard or extended SOHND excluding level $\mathrm{V}$ would be acceptable in the treatment of patients with $\mathrm{cN} 1$ oral cancers.

It should be noted that false-positive and false-negative rates of preoperatively diagnosed clinical lymphnode metastasis were relatively higher in the previous studies [5, 7]. Given the recent advances in the accuracy of diagnostic modalities such as US-guided FNAB and SNB, further research needs to be done in more strictly defined N1 (level I) patients. From the oncological point of view, survival rate of the patients with $\mathrm{cN} 1 / \mathrm{pN} 2 \mathrm{~b}$ was unfavorable in the present study. Eight out of 29 patients with $\mathrm{pN} 2 \mathrm{~b}$ died of disease during the observation period. In the patients with clinically $\mathrm{N} 1$ and multiple occult histopathological metastases, the tumor may be high biological malignancy. Postoperative treatments, such as chemotherapy, radiotherapy, and/or immunotherapy, should be reconsidered, to control the primary tumor as well as regional and distant metastases for these patients.

Another limitation of this study is the TNM staging system employed in the analysis. Since we could not obtain the precise value of depth of invasion (DOI) of early tongue cancers in old cases, we staged according to the seventh TNM staging system, instead of eighth. Thus, we did not evaluate the correlation between DOI and metastasis to level III or IV in this analysis. Although there were no significant differences in the frequency of metastasis to level III or level IV according to T classification in the present study, multi-institutional prospective study using eighth TNM staging system [20] should be performed to draw more definitive conclusions.

Avoiding level IV dissection has several potential merits to prevent additional operation time and/or blood loss, chylorrhea, and phrenic nerve paralysis when less-experienced surgeons perform dissection of level IV. However, these merits are small for well-experienced surgeons. In addition, our previous studies have found no significant difference between levels I-III and I-IV dissections in terms of postoperative quality of life (QOL) and shoulder function, provided that neck rehabilitation was performed after surgery [19]. Besides the extent of dissection, providing effective postoperative rehabilitation is also an important issue.

\section{Conclusions}

In conclusion, none of the 100 patients diagnosed as $\mathrm{cN} 1$ oral cancer had pLN to level V, suggesting that level V dissection can be excluded. Metastasis to level IV was observed only in two patients with tongue cancer who had a suspected metastasis at level II before surgery, both being pN2b subjects. SOHND is not necessarily an appropriate option for a $\mathrm{cN} 1$ patient with oral cancer when the cancer originates in the tongue, the patient has a suspicious lymph node at level II before surgery, and/or intraoperative diagnosis finds multiple lymph-node metastases. Further investigation with more patients is needed to find the range of patients for whom SOHND is indicated.

Funding This work was partly supported by Japan Agency for Medical Research and Development under Grant number 18ck0106223h0003. 


\section{Compliance with ethical standards}

Conflict of interest The authors have no conflict of interest to disclose regarding this article.

Open Access This article is licensed under a Creative Commons Attribution 4.0 International License, which permits use, sharing, adaptation, distribution and reproduction in any medium or format, as long as you give appropriate credit to the original author(s) and the source, provide a link to the Creative Commons licence, and indicate if changes were made. The images or other third party material in this article are included in the article's Creative Commons licence, unless indicated otherwise in a credit line to the material. If material is not included in the article's Creative Commons licence and your intended use is not permitted by statutory regulation or exceeds the permitted use, you will need to obtain permission directly from the copyright holder. To view a copy of this licence, visit http://creativecommons.org/licenses/by/4.0/.

\section{References}

1. Ferlito A, Som PM, Rinaldo A et al (2000) Classification and terminology of neck dissections. ORL J Otorhinolaryngol Relat Spec 62:212-216

2. Shah JP, Candela FC, Poddar AK (1990) The patterns of cervical lymph node metastases from squamous carcinoma of the oral cavity. Cancer 66:109-113

3. Brazilian Head and Neck Cancer Study Group (1998) Results of a prospective trial on elective modified radical classical versus supraomohyoid neck dissection in the management of oral squamous carcinoma. Am J Surg 176:422-427

4. Fasunla AJ, Greene BH, Timmesfeld N et al (2011) A meta-analysis of the randomized controlled trials on elective neck dissection versus therapeutic neck dissection in oral cavity cancers with clinically node negative neck. Oral Oncol 47:320-324

5. D'Cruz AK, Vanish R, Kapre N et al (2015) Elective versus therapeutic neck dissection in node-negative oral cancer. N Engl J Med 373:521-529

6. Schilling C, Shaw R, Schache A et al (2017) Sentinel lymph node biopsy for oral squamous cell carcinoma. Where are we now? $\mathrm{Br}$ J Oral Maxillofac Surg 55:757-762

7. Kowalski LP, Carvalho AL (2002) Feasibility of supraomohyoid neck dissection in N1 and N2a oral cancer patients. Head Neck 128:1180-1184

8. Asakage T, Kishimoto S, Saikawa M et al (2005) The standardization of neck dissection for tongue carcinoma. Toukeibu Gan 31(4):536-540 (in Japanese)
9. Sun R, Tang X, Yang Y et al (2015) (18)FDG-PET/CT for the detection of regional nodal metastasis in patients with head and neck cancer: a meta-analysis. Oral Oncol 51(4):314-320

10. Liao LJ, Lo WC, Hsu WL et al (2012) Detection of cervical lymph node metastasis in head and neck cancer patients with clinically N0 neck-a meta-analysis comparing different imaging modalities. BMC Cancer 12:236

11. Andersen PE, Warren F, Spiro J et al (2002) Results of selective neck dissection in management of the node-positive neck. Arch Otolaryngol Head Neck Surg 128(10):1180-1184

12. Suzuki M, Yoshino K, Fujii T et al (2009) Appropriate area of neck dissection for N1 tongue cancer. Toukeibu Gan 35(4):370 373 (in Japanese)

13. Harada H, Omura K, Shimamoto H (2014) Selective neck dissection for oral squamous cell carcinoma. Toukeibu Gan 40(1):16-22 (in Japanese)

14. Shimbashi W, Kawabata K, Mitani H et al (2012) Appropriate area of selective neck dissection for tongue carcinoma. Toukeibu Gan 38(4):425-429 (in Japanese)

15. Dias FL, Lima RA, Kligerman J et al (2006) Relevance of skip metastases for squamous cell carcinoma of the oral tongue and the floor of the mouth. Otolaryngol Head Neck Surg 134(3):460-465

16. Koerdt S, Röckl J, Rommel N et al (2016) Lymph node management in the treatment of oral cancer: analysis of a standardized approach. J Craniomaxillofac Surg 44(10):1737-1742

17. Liang L, Zhang T, Kong Q et al (2015) A meta-analysis on selective versus comprehensive neck dissection in oral squamous cell carcinoma patients with clinically node-positive neck. Oral Oncol 51:1076-1081

18. Cariati P, Cabello Serrano A, Fernandez Solis J et al (2018) Distribution of cervical metastasis in tongue cancer: are occult metastases predictable? A retrospective study of 117 oral tongue carcinomas. J Craniomaxillofac Surg 46(1):155-161

19. Inoue $\mathrm{H}$, Nibu $\mathrm{K}$, Saito $\mathrm{M}$ et al (2006) Quality of life after neck dissection. Arch Otolaryngol Head Neck Surg 132(6):662-666

20. Ridge JA, Lydiatt WM, Patel SG et al (2017) Part II 7. Lip and oral cavity. In: Avin MB (ed) AJCC cancer staging manual, 8th edn. Springer, Switzerland, pp 79-94

Publisher's Note Springer Nature remains neutral with regard to jurisdictional claims in published maps and institutional affiliations. 